\title{
Protocol Heterogeneity Issues of Incremental High-Density Wi-Fi Deployment
}

\author{
Haymanot Gebre-Amlak, Md Tajul Islam, Daniel Cummins, Mohammed Al \\ Mansoori, Baek-Young Choi \\ Email: \{hhgc77, mi8rd, cumminsdm, mkakh3, choiby\}@umkc.edu \\ Department of Computer Science and Electrical Engineering, \\ University of Missouri-Kansas City, \\ 5100 Rockhill Rd. Kansas City, MO, USA
}

\begin{abstract}
Going beyond the traditional coverage-oriented Wi-Fi network design, the recent Wi-Fi networks are designed for high traffic demand with high density deployments. A university campus environment is particularly unique in that a large number of users with multiple heterogeneous devices demand high capacity and performance from a wireless network over a wide geographical area. From a network management perspective, not only should the network support heterogeneous Wi-Fi protocols and devices, but high-density access points (APs) are needed to handle the high traffic demands. To meet the rising demands WiFi AP upgrades are deployed incrementally over an extended period to cover the vast area found in a campus setting, which is different from a building-level Wi-Fi network.

In this paper, we present a measurement study to bring forth wireless network management issues faced during incremental Wi-Fi deployment on a university campus network. We discuss various design considerations given to incremental deployments of Wi-Fi 802.11 (ac) including replacing older Wi-Fi versions, and addressing compatibility, data rate, coverage, and performance concerns. In addition, we perform pre-and-post upgrade evaluations using different network performance analysis tools. This study will shed light on heterogeneous large-scale Wi-Fi network management issues, as these will become applicable with the increasing prevalence of large metro area wireless networks.
\end{abstract}

\section{Introduction}

The availability of versatile and resilient wireless networks with high-speed performance and Wi-Fi on-the-go service with always-connected features for bandwidthintensive applications is considered a basic necessity these days. Faster connection and speedy performance are the bare requirements for emerging bandwidth intensive services like high resolution video uploading (i.e., YouTube), video streaming (i.e., Netflix), virtual reality, augmented reality, real-time updating data (i.e., Facebook), online gaming, and live stream video (i.e. surveillance camera data). For example, Cisco announced [11 that it is expecting the global 
traffic to extend to $3.3 \mathrm{ZB}$ per year by 2021 or $278 \mathrm{~EB}$ per month 11 . Devices and connections are growing at a 10 percent compound annual growth rate (CAGR) [5], and North American growth is expected to be the highest rate in the world $[11]$. Adaptation of IPv6 throughout the Internet allows for an effortless interaction among the Internet of Things (IoT) 12. Internet traffic is increased by internet video, video-streaming, gaming, video-conferencing, and video specific applications. Most internet users use the internet on their mobile devices such as tablets, phone, laptop, etc., and they get their connection through wireless data services. They require high throughput and seamless connectivity without outage from their network services. IEEE 802.11 [25] wireless network standards,

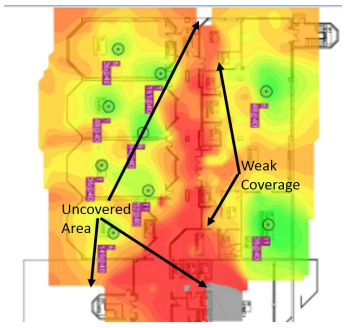

Fig. 1. An Example of Coverage Map

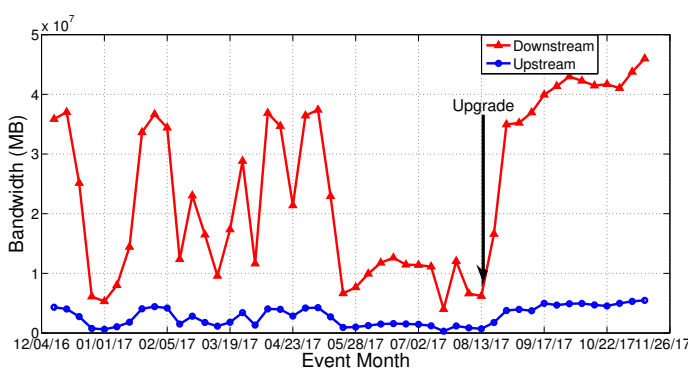

Fig. 2. Network Traffic

which is the most publicly used, have evolved to meet these growing requirements in terms of features like extended channel binding, multi-user Multiple In Multiple Out (MIMO) 26 ability, wide range of modulation, beam-forming, MAC modification, coexistence mechanisms, throughput and much more 16 .

University Wi-Fi network accommodate thousands of mobile and wireless devices being used on a campus network. Multiple features of network utilization such as device variation, bandwidth requirements, application variability, operating system variability, user authentication and data off-loading, can be observed in a campus network. Each university strives to provide the best network service to its users despite the challenge of a fixed budget. To support the growing demand, it was necessary to upgrade the Wi-Fi network to improve coverage area, network speed, and throughput. The network team has taken two major steps for this enhancement. First, increase the number of access points (APs) to address the coverage gap. Second, upgrade the Wi-Fi network to 802.11 (ac) protocol to increase performance. In this paper, we conduct an extensive measurement analysis of the Wi-Fi network condition throughout the access layer of a university campus network, the University of Missouri - Kansas City (UMKC). We found some interesting phenomena like the variety of user density in the different locations with diversified bandwidth demands, lacking coverage in some area resulting in coverage gap, data rate degradation in certain points, and protocol compatibility issues. Figure 1 represents the signal strength and 
coverage map of a floor in a campus building prior to the upgrade. Furthermore, our observation indicates that between December 2016 and October 2017, the networks traffic (upstream and downstream) has increased by up to $20 \%$, even though the number of clients has decreased, as illustrated in Figure 2.

The remainder of the paper is organized as follows. Section 2 discusses the related work. Section 3 outlines the deployment consideration and design. Section 4 presents our evaluation of measurement and analysis. We conclude the paper in Section 5 .

\section{Related Work}

\subsection{Wi-Fi Performance}

Performance measurement analysis research done by 24 on IEEE 802.11 (ac) Wi-Fi in a large 24-node indoor test-bed reveals the impact of channel width, network deployment density, and type/volume of traffic on the achieved network performance in dense indoor deployments 27. A Framework of Heterogeneous Network(HetNet) for Mobile cloud computing (MCC) has been introduced by 21, and they discuss the challenges for supporting MCC applications in HetNet. A focused study of dual-band APs performance in heterogeneous client adapter was done in 14 with a goal to understand the characteristics of 802.11 (n) networks in heterogeneous deployments. Their result reveals that dual band routers can effectively help in reducing the negative effects of adapters heterogeneity as well as providing backward compatibility.

Wi-Fi network supports varying levels of performance, depending on the technology standard. Research 22 introduces a key mandatory and optional PHY features, as well as the MAC enhancements of 802.11 (ac) over the existing 802.11 (n) standard in the evolution towards higher data rates. They compare the MAC performance between 802.11 (ac) and 802.11 (n) over three different frame aggregation mechanisms, viz., aggregate MAC service data unit (A-MSDU), aggregate MAC protocol data unit (A-MPDU), and hybrid A-MSDU/A-MPDU aggregation through numerical analysis and simulations. The result showed 802.11 (ac) outperforms 802.11 (n) with maximum throughput by $28 \%$ (84\%).

\subsection{Wi-Fi Usage}

Study on the usage of the Google Wi-Fi network deployed in Mountain View, CA has been done by 15. Their finding showed that usage naturally falls into three categories based almost entirely on client device type, traditional laptop

users, fixed-location access devices, and PDA-like smart-phone devices and they have a diverse set of mobility patterns that map well to the archetypal use cases for traditional access technologies. In a study done by [19, for mobile clients to evaluate and select both APs and spectral bands in wide-spectrum networks that provide access to four bands at $700 \mathrm{MHz}, 900 \mathrm{MHz}, 2.4 \mathrm{GHz}$ and $5 \mathrm{GHz}$ by a single operational access network. Their finding showed under a diverse set 
of operating conditions, mobile clients can accurately predict their performance without a direct measurement at their current location and spectral bands. The challenge to provide seamless mobility emerges as a key topic in various standardization bodies as explored by 18 discusses the support of seamless handover between homogeneous networks. The continued effort in pursuit of gigabit wireless communications has been most noticeable in the IEEE 802.11 WLAN 20] in recent years. In 2010, the Wireless Gigabit (WiGig) Alliance, formed by a consortium of industry leaders, has completed the defined first draft of a unified architecture to enable tri-band communications over the frequency bands of 2.4, 5, and $60 \mathrm{GHz}$ in their WiGig specification 23.

Our research differs from these authors; we focused on usage behavior measurement study of the access layer to bring light to the management issue discovered during incremental Wi-Fi deployment. We analyze less known network management issues such as coverage gap, bandwidth overload, and association/authentication failures due to roaming in a campus network.

Note that while there are a number of Wi-Fi measurement works available little studies have been done to provide insight on network management issues resulting from incremental, heterogeneous high-density 8 Wi-Fi deployment.

\section{Wi-Fi Deployment Design}

Incremental deployments were done over four years period. How to design heterogeneous compatibility, data rate, coverage, and performance were factored into the deployment plan and roll-out schedules [17].

Table 1. Data Sets Used

\begin{tabular}{|c||c|c|}
\hline Data Source & Data Size & Duration \\
\hline \hline Syslog & 15.3 billion records & Jan 2014 - Jan 2018 \\
\hline Cisco Prime & (14 categories)/(126 items) report & Oct 2016 - Jan 2018 \\
\hline Ekahau Survey Map & $\begin{array}{c}\text { 41 Buildings Blue Print \& } \\
\text { Signals of 1137 Access Points }\end{array}$ & Jan 2014 - Jan 2018 \\
\hline
\end{tabular}

Table 2. Data Source and Tools used for Analyzing Coverage, Authentication and Bandwidth Issues

\begin{tabular}{|c||c||c||c|}
\hline Issues & Coverage Gaps & Authentication Failure & BW Overload \\
\hline \hline Analysis & Ekahau & Splunk \& Syslog & Cisco Prime \& WLC \\
\hline Data Sources & Survey Map \& Syslog & Netflow & Radius Logs \\
\hline Tools & Ekahau \& Splunk & Cisco Prime & Ekahau \\
\hline
\end{tabular}




\subsection{Analysis Tool}

Wireless devices need to be compatible with most of the wireless protocols and decide to select the best channel and wireless protocol based on wide range of parameters. The upgrade had to be backward compatible to accommodate heterogeneous devices [27. The University Information Services department use network log events with Splunk and Ekahau Spectrum Analyzers to locate the coverage gaps through out the campus Wi-Fi network. There were many errors from re-authentication failure, and co-channel interference due to the wide range of the $2.4 \mathrm{GHz}$ APs. Additionally, Wi-Fi network faced bandwidth overload in most of the $2.4 \mathrm{GHz}$ APs due to the extensive coverage area which reaches too many clients far more than the APs' capacity. Deploying an upgrade to replace the older APs which supported $802.11(\mathrm{~b} / \mathrm{g} / \mathrm{n})[13$ with newer APs that supports both $802.11(\mathrm{~b} / \mathrm{g} / \mathrm{n} / \mathrm{ac})$ and the adaptive 802.11 (r) [3] with FT feature would address these rising concerns. There are several tools used for the analysis of the upgrade and the different issues resolved by the upgrade as listed in Table 2 . Table 1 discusses the data set used for our analysis. We had 15.3 billion records generated by Syslog server over four years period. The second data source is Cisco Prime consisting of $1 \mathrm{hr}, 1$ week, 1 month and/or 1 year reports data from 14 different Categories, Autonomous AP, Clean Air, Clients, Compliance, Composite, Device, Guests, Identity Service Engin, Mesh, Network Summary, Performance, Raw NetFlow, Security and System Monitoring. There are 126 items reports under these categories.

\subsection{Channel Planning and Band Select Feature}

Several questions came to mind while planning the Wi-Fi protocol. What channels do the heterogeneous devices in the campus network support? Many older devices may not support the Extended channels and when that happens, the upgrade should only be providing redundant coverage. What is the student capacity in each class rooms? High density deployments were needed in some of the large auditoriums, which required increasing the number of APs in addition to the Wi-Fi upgrade. Consideration was given for APs to be allocated where coverage is optimized with minimum number of APs. Clients inside a pair of APs need to be within coverage distance from at least one of APs.

The $2.4 \mathrm{GHz}$ band covers a larger range than $5 \mathrm{GHz}$ and is commonly used on the campus network. Cisco APs by default send quicker probe response from $2.4 \mathrm{GHz}$ band, resulting in more connection to $2.4 \mathrm{GHz}$ band until the AP is overloaded and can no longer accept new connections. To overcome this crowding issue, Cisco introduced a feature called 'Band Select' [4, which allows dual-band clients to prefer the $5 \mathrm{GHz}$ band over the $2.4 \mathrm{GHz}$. Band selection works by regulating probe responses to clients by making $5 \mathrm{GHz}$ channels more attractive to dual-band clients from delaying $2.4 \mathrm{GHz}$ probe responses. For example, the number of device association that is higher than $\mathrm{N}$ (our network set $\mathrm{N}$ to be 12) will have $2.4 \mathrm{GHz}$ response suppressed $\mathrm{M}$ (our network set $\mathrm{M}$ to be 4 ) times before allowing the clients to receive Probe Response from $2.4 \mathrm{GHz}$ wireless 
band. This may cause a small delay for non-dual band $2.4 \mathrm{GHz}$ only devices, however, will alleviate the crowding issue of $2.4 \mathrm{GHz}$ wireless band and send dual-band clients to $5 \mathrm{GHz}$ wireless band creating a more balanced network.

\subsection{Addressing Roaming Issue}

Wi-Fi 802.11 (ac) has the fastest data rate, 1300 Megabits per second (Mbps) and compared to 802.11 (n) 25] typically $450 \mathrm{Mbps}$, it is 3x faster. Fast Transition(FT) [1, a feature of 802.11 (ac), makes roaming between two adjacent 5 $\mathrm{GHz}$ band seamless. However, if a client roams outside of 802.11 (ac) or even between two adjacent $5 \mathrm{GHz}$ band and $2.4 \mathrm{GHz}$ band, there is bound to be a delay. FT feature, included in the Wi-Fi deployment, permit continuous connectivity aboard wireless devices in motion, with fast and secure hand-offs from one base station to another while the client is roaming. FT will not be explored in this work. However, it will be scoped as part of future works.

\subsection{Managing Coverage Gap}

It is part of the design process to locate the holes in the existing Wi-Fi coverage area in order to identify the precise locations to place the new APs to provide the ultimate coverage. The initial installation had the APs stacked in one location of the building providing inefficiency and weak coverage to no coverage outside of the stacked APs coverage area. The current design fans out these APs to maximize the coverage area with minimum number of APs. Additional APs were placed throughout the campus locations to manage the load capacity for high-density areas.

Table 3. 802.11 (ac) Wireless Upgrade Summary

\begin{tabular}{|c|c|c|}
\hline Model & Desc & AP Count \\
\hline \hline 1810 & 802.11 (ac) Wave 2 MU-MIMO & 735 \\
\hline $3802 \mathrm{I}$ & $\begin{array}{c}\text { decisions based on } \\
\text { Wave 1 end-device activities }\end{array}$ & 65 \\
\hline 3702 & Wave 1 150 Mbps & 199 \\
\hline 3602 Radio & $\begin{array}{c}\text { dual-band 2.4/5-GHz } \\
\text { - Wave 1 integrated radios }\end{array}$ & 130 \\
\hline
\end{tabular}

\subsection{Wi-Fi Upgrade Summary}

The incremental Wi-Fi upgrade targeted the wireless infrastructure of several buildings including the university dormitories, and an off-campus network. There were 1187 APs upgraded to 802.11 (ac). Several of the old APs were replaced 
by the new APs with updated features. Majority of the APs (735), had Model 1810 Wave 2 with feature MU-MIMO 26. MU-MIMO provides concurrent downstream communications to multiple wireless devices allowing client devices to get on and off the network faster, enabling more clients to use the network. 199 of the APs were with Model 3702 Wave 1 dual-band radios with a data rate of 150 Mbps. Table 3 lists the model and the number of AP which were deployed over the summer/winter weeks targeting minimal impact to clients.

\section{Evaluation}

In this section we perform pre-and-post upgrade evaluation using different network performance analysis tools. Cisco Prime Infrastructure reporting $[9]$ is a tools used by network team to help monitor the system and network health and is used for troubleshooting network problems. To evaluate the sheer volume of Syslog messages generated from the Wi-Fi network during the upgrade period between 2015 and 2018, we use Big Data analytic tool named Splunk. We used Ekahau Wi-Fi Site Survey tool to capture the coverage map before and after the deployment. We used Wireshark to capture the packets of the roaming clients and FT authentications. We also used Wireless LAN Controller (WLC) and Radius logs to gather the Wi-Fi traffic activities.

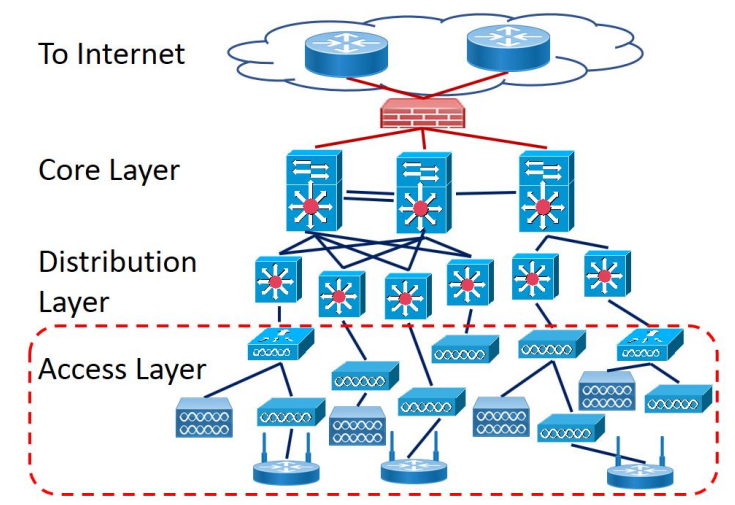

Fig. 3. Campus network architecture with three layers: core, distribution, and access layers

\subsection{Campus Network Setup}

The campus network is hierarchical with three layers which is a common practice for campus or enterprise networks, illustrated by Figure 3. UMKC network's core routing depends mainly on two core routers, one as primary and the second as 
fail-over and load-balance. There is a third core router that is dedicated to the University housing and eduroam network. The second or middle layer is the distribution layer, connects the core layer (using routers) to the access layer (using switches). The lower layer includes all the edge switches, and APs which connect to the end users. The Wi-Fi network extends from our main university campus to nearby campus in downtown Kansas City, to Union Station of Kansas City, which is about 15 miles away. Within our university network, there are many networks, each serving a specific service. UMKCWPA is the main SSID for the UMKC wireless network. The eduroam is the network SSID for the educational network access for all the eduroam $[6]$ parties. eduroam (education roaming) is an international roaming service for users in research, higher education and further education. It provides a single authentication access for all the mobile connectivity requirements of an institution across 78 countries in thousands of locations. The Wi-Fi network is primarily accessed by students, faculty, staff. Guest accounts use the guest network, which is dedicated for guests with very limited temporary access. The media network is the network that connects the media devices such as ROKUs [10], Firesticks [7, Chromecast [2], and Digital Video Recorder (DVR) boxes.
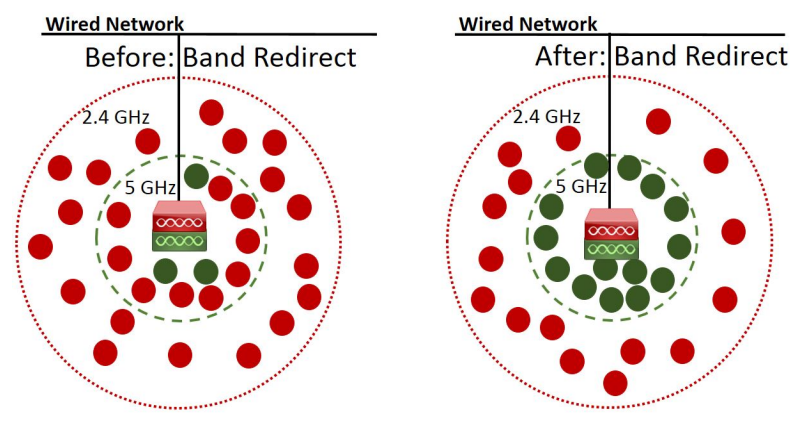

Fig. 4. Impact of Band Redirection

\subsection{Client Redirection of $2.4 \mathrm{GHz}$ to $5 \mathrm{GHz}$ Band}

$5 \mathrm{GHz}$ band offers a lot more space compared to $2.4 \mathrm{GHz}$, though it is not always fully utilized. Figure 4 illustrates authentication before and after the Band Select Feature suppresses client's association request to $2.4 \mathrm{GHz}$ multiple times guiding the requests to $5 \mathrm{GHz}$ band. We can see that "Before: Band Redirect" most clients where connecting to $2.4 \mathrm{GHz}$ band by default. However, "After: Band Redirect", the dual band clients are guided toward the $5 \mathrm{GHz}$ band. Our campus analysis indicated that there we more $5 \mathrm{GHz}$ band authentication than $2.4 \mathrm{GHz}$ as most laptops are only $5 \mathrm{GHz}$ enabled. Between October 2016 and 
2017 we observe the gradual decline in use of $2.4 \mathrm{GHz}$ band and increase in $5 \mathrm{GHz}$ band as illustrated by Figure 6. Each dot on the graph represents the aggregated authentication count for a week. The lower peaks are due to holiday seasons, such as season break where student attendance is low. Comparing the first five weeks of the graph with the last five weeks, we can see that the client using $5 \mathrm{GHz}$ band increased by up to 1150 count a week while clients using 2.4 $\mathrm{GHz}$ band decreased by up to 750 counts a week.

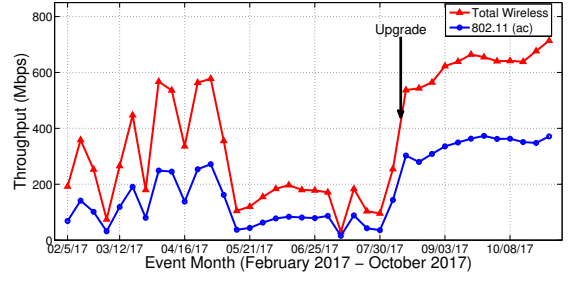

Fig. 5. 802.11 total vs $802.11(\mathrm{ac})$ Throughput

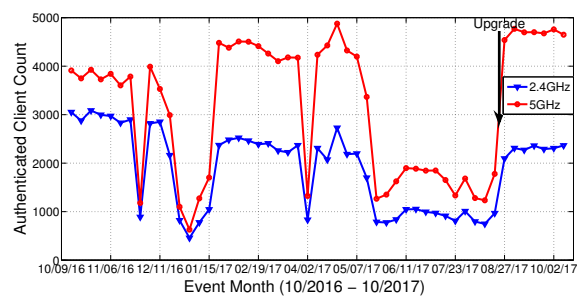

Fig. 6. $2.4 \mathrm{GHz}$ vs $5 \mathrm{GHz}$ Authentication

\subsection{Throughput: Total 802.11 vs 802.11 (ac)}

There were several activities during the last year that impacted the throughput to fluctuate during the Spring semester. The week of February 27th the WiFi network faced brief outage causing a dip in the traffic report. However, the controller/fail-over instantly corrected itself and traffic resumed. The week of April 2nd following spring break, our campus network upgraded to a new router resulting in short interruption. The third dip during April 23rd was due to performance issue which caused the router to reboot. Total Wireless network vs. 802.11 (ac) illustrated by Figure 5 shows the weekly aggregated throughput in $\operatorname{Mbps}$ (Mega bit per second) between February 2017 and October 2017, with an increase in throughput for both Wi-Fi 802.11 (ac) and Total wireless (Wi-Fi 802.11) between spring and fall semester. Each dot on the graph represents the value of aggregated throughput for one week. Shortly after the Wi-Fi upgrade, throughput increased for both Wi-Fi 802.11 as a whole and 802.11(ac).

\subsection{Coverage Gap}

After the recent implementation in January 2018 which included installation of several new APs to provide coverage to the red zone, the coverage improved significantly. Figure 7 represents the initial coverage with 9 APs in 2014, where the majority of the area was red and orange indicating coverage gaps. Figure 8 illustrates the coverage map in 2016 after 2 additional APs were installed and coverage showed improvement. Figure 9, recent coverage representation January 2018, with total of 19 APs and majority of them were dual band high-density. 


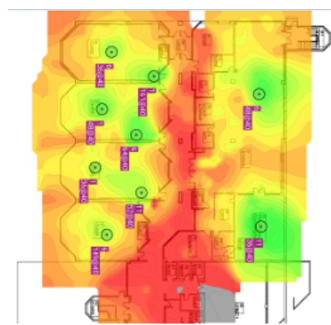

Fig. 7. Coverage in 2014

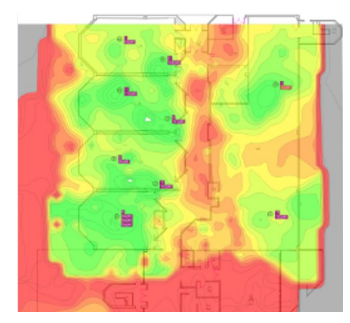

Fig. 8. Coverage in 2016

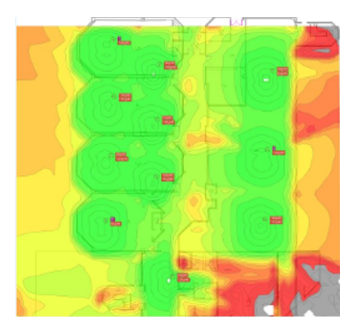

Fig. 9. Coverage 2018

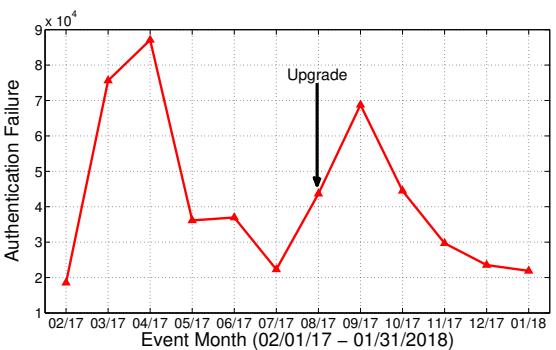

Fig. 10. Authentication Failure Due to Roaming

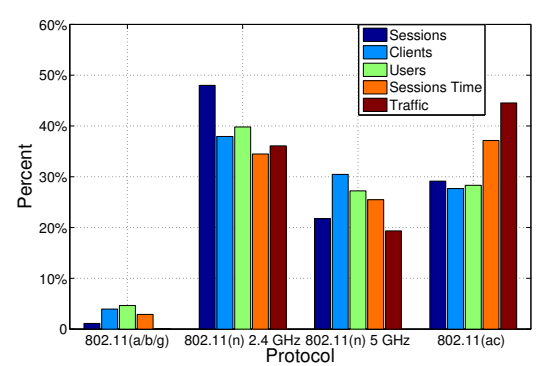

Fig. 11. Usage Summary by Protocol

\subsection{Authentication Failure Due To Roaming}

Figure 10 illustrates authentication failure due to roaming between February 2017 and January 2018. The highest failure event in the spring semester happened in April with the total of $87 \mathrm{~K}$ messages. In the fall semester, the peak month was September total failure message of $68.7 \mathrm{~K}$, showing a decrease of $18 \mathrm{~K}$ compared to the spring semester. The upgraded feature enables fast and smooth roaming transition between 802.11 (ac), decreasing the authentication failure caused by roaming clients.

\subsection{Heterogeneous Protocols}

Figure 11 illustrates the percentage of all the protocols used in the campus network over a 31 days period grouped by sessions, clients, users, session time and traffic. Although, the top three protocols were 802.11 (n) $2.4 \mathrm{GHz}, 802.11$ (ac) and 802.11 (n) $5 \mathrm{GHz}$ in respective order. Protocol 802.11 (ac) shows the highest Session to Traffic ratio and the most Session Time with the least clients. Protocols 802.11 (a/b/g) are the least used protocol in the campus network. However, the campus network has to support these heterogeneous protocols to provide the environment for all devices needing Wi-Fi access. 


\section{Conclusions and Future Work}

We have conducted extensive analysis of heterogeneity issues encountered during an incremental campus Wi-Fi protocol deployment. We have explored various design considerations for heterogeneous compatibility, data rate, coverage, and performance. Furthermore, we discussed the issues around coverage gap, load balance, and roaming. This study of a campus incremental Wi-Fi deployment provides insights on the behaviors of Wi-Fi access network availability, and potential end-to-end expectations for high-speed and bandwidth-intensive clients. After the deployment, the most traffic is generated by 802.11 (ac) protocol. However, heterogeneous protocols such as $802.11(\mathrm{a} / \mathrm{b} / \mathrm{g} / \mathrm{n})$ still need to coexist until all devices are 802.11 (ac) compatible.

In the future, we plan to explore fast transition, performance enhancement, power saving, and time improvement benefits gained from upgrading to Wi-Fi 802.11 (ac) protocol.

\section{References}

1. 802.11r Vulnerability (CVE: 2017-13082) FAQ. https://documentation.meraki. com/zGeneral_Administration/Support/802.11r_Vulnerability_(CVE\%3A_

2017-13082)_FAQ. Accessed: 2017-10.

2. Chromcast, howpublished $=$ https://store.google.com/product/chromecast_ 2015, note $=$ Accessed: 2018-03.

3. Cisco 802.11r. https://www.cisco.com/c/en/us/td/docs/wireless/ controller/8-1/Enterprise-Mobility-8-1-Design-Guide/Enterprise_ Mobility_8-1_Deployment_Guide/Chapter-11.html. Accessed: 2017-10.

4. Cisco Band Select. https://www.cisco.com/c/en/us/td/docs/switches/lan/ catalyst3850/software/release/3se/system_management/configuration_ guide/b_sm_3se_3850_cg/b_sm_3se_3850_cg_chapter_0110.html. Accessed: 2018-02.

5. Compound Annual Growth Rate - CAGR. http://www . investopedia.com/terms/ c/cagr.asp Accessed: 2017-10.

6. eduroam blog. https://www.eduroam.org/?p=where Accessed: 2017-10.

7. FireStick, howpublished = https://www.amazon.com/ amazon-fire-tv-stick-with-alexa-voice-remote-streaming-media-player/ dp/b00zv9rdkk, note $=$ Accessed: 2018-03.

8. High-Density Wi-Fi Design Principles. https://media.aerohive.com/documents/ 2034844328_Aerohive-Whitepaper-Hi-Density_Principles.pdf Accessed: 2017-09.

9. Prime Report. https://www.cisco.com/c/en/us/td/docs/net_mgmt/prime/ infrastructure/3-0/user/guide/pi_ug/reps.html accessed: 2017-10.

10. Roku, howpublished $=$ https://www.roku.com/, note $=$ Accessed: 2018-03.

11. The Zettabyte Era: Trends and Analysis. https://www.cisco.com/c/en/us/ solutions/collateral/service-provider/visual-networking-index-vni/ vni-hyperconnectivity-wp.html Accessed: 2017-10.

12. What is IoT? http://internetofthingsagenda.techtarget.com/definition/ Internet-of-Things-IoT. Accessed: 2017-10. 
13. Next generation ieee 802.11 wireless local area networks. Comput. Commun., 75(C):1-25, February 2016.

14. Murad A. Abusubaih, Saif Najem Eddin, and Ahmad Khamayseh. Ieee 802.11n dual band access points for boosting the performance of heterogeneous wifi networks. In Proceedings of the 8th ACM Workshop on Performance Monitoring and Measurement of Heterogeneous Wireless and Wired Networks, PM2HW2N '13, pages 1-4, New York, NY, USA, 2013. ACM.

15. M. Afanasyev, T. Chen, G. M. Voelker, and A. C. Snoeren. Usage patterns in an urban wifi network. IEEE/ACM Transactions on Networking, 18(5):1359-1372, Oct 2010.

16. C. T. Chou, A. Misra, and J. Qadir. Low-latency broadcast in multirate wireless mesh networks. IEEE Journal on Selected Areas in Communications, 24(11):20812091, Nov 2006.

17. A. Dubey and J. Hudepohl. Towards global deployment of software engineering tools. In 2013 IEEE 8th International Conference on Global Software Engineering, pages 129-133, Aug 2013.

18. Marc Emmelmann, Sven Wiethoelter, Andreas Koepsel, Cornelia Kappler, and Adam Wolisz. Moving toward seamless mobility: state of the art and emerging aspects in standardization bodies. Wireless Personal Communications, 43(3):803816, Nov 2007.

19. A. Giannoulis, P. Patras, and E. W. Knightly. Mobile access of wide-spectrum networks: Design, deployment and experimental evaluation. In 2013 Proceedings IEEE INFOCOM, pages 1708-1716, April 2013.

20. G. R. Hiertz, D. Denteneer, L. Stibor, Y. Zang, X. P. Costa, and B. Walke. The ieee 802.11 universe. IEEE Communications Magazine, 48(1):62-70, January 2010.

21. L. Lei, Z. Zhong, K. Zheng, J. Chen, and H. Meng. Challenges on wireless heterogeneous networks for mobile cloud computing. IEEE Wireless Communications, 20(3):34-44, June 2013.

22. Eng Hwee Ong, J. Kneckt, O. Alanen, Z. Chang, T. Huovinen, and T. Nihtil. Ieee 802.11ac: Enhancements for very high throughput wlans. In 2011 IEEE 22nd International Symposium on Personal, Indoor and Mobile Radio Communications, pages 849-853, Sept 2011.

23. Ali S. Sadri. Defining the future of multi-gigabit mmwave wireless communications. In Proceedings of the 2010 ACM International Workshop on mmWave Communications: From Circuits to Networks, mmCom '10, pages 1-2, New York, NY, USA, 2010. ACM.

24. L. Simi, J. Riihijrvi, and P. Mhnen. Measurement study of ieee 802.11ac wi-fi performance in high density indoor deployments: Are wider channels always better? In 2017 IEEE 18th International Symposium on A World of Wireless, Mobile and Multimedia Networks (WoWMoM), pages 1-9, June 2017.

25. Chih-Yu Wang and Hung-Yu Wei. Ieee 802.11n mac enhancement and performance evaluation. Mob. Netw. Appl., 14(6):760-771, December 2009.

26. Jie Xiong, Karthikeyan Sundaresan, Kyle Jamieson, Mohammad A. Khojastepour, and Sampath Rangarajan. Midas: Empowering 802.11ac networks with multipleinput distributed antenna systems. In Proceedings of the 10th ACM International on Conference on Emerging Networking Experiments and Technologies, CoNEXT '14, pages 29-40, New York, NY, USA, 2014. ACM.

27. A. Zubow and R. Sombrutzki. Adjacent channel interference in ieee 802.11n. In 2012 IEEE Wireless Communications and Networking Conference (WCNC), pages 1163-1168, April 2012. 